\title{
The circumstellar envelope of IRC+10216 from milli-arcsecond to arcmin scales ${ }^{\star}$
}

\author{
I. C. Leão ${ }^{1,2}$, P. de Laverny ${ }^{1}$, D. Mékarnia ${ }^{1}$, J. R. De Medeiros ${ }^{2}$, and B. Vandame ${ }^{3}$ \\ 1 Observatoire de la Côte d'Azur, Dpt Cassiopée, CNRS-UMR 6202, BP 4229, 06304 Nice Cedex 4, France \\ e-mail: [leao; laverny; mekarnia]@obs-nice.fr \\ 2 Departamento de Física, Universidade Federal do Rio Grande do Norte, 59072-970 Natal, RN, Brazil \\ ${ }^{3}$ European Southern Observatory, Karl-Schwarzschild-Str. 2, 85748 Garching b. München, Germany \\ Received 23 November 2005 / Accepted 15 January 2006
}

\section{ABSTRACT}

\begin{abstract}
Aims. Analysis of the innermost regions of the carbon-rich star IRC+10216 and of the outer layers of its circumstellar envelope have been performed in order to constrain its mass-loss history.

Methods. We analyzed the high dynamic range of near-infrared adaptive optics and the deep $V$-band images of the circumstellar envelope of IRC+10216 using high angular resolution, collected with the VLT/NACO and FORS1 instruments.

Results. From the near-infrared observations, we present maps of the sub-arcsecond structures, or clumps, in the innermost regions. The morphology of these clumps is found to strongly vary from $J$ - to $L$-band. Their relative motion appears to be more complex than proposed in earlier works: they can be weakly accelerated, have a constant velocity, or even be motionless with respect to one another. From $V$-band imaging, we present a high spatial resolution map of the shell distribution in the outer layers of IRC+10216. Shells are resolved well up to a distance of about $90^{\prime \prime}$ to the core of the nebula and most of them appear to be composed of thinner elongated shells. Finally, by combining the NACO and FORS1 images, a global view is present to show both the extended layers and the bipolar core of the nebula together with the real size of the inner clumps.

Conclusions. This study confirms the rather complex nature of the IRC+10216 circumstellar environment. In particular, the coexistence at different spatial scales of structures with very different morphologies (clumps, bipolarity, and almost spherical external layers) is very puzzling. This confirms that the formation of AGB winds is far more complex than usually assumed in current models.
\end{abstract}

Key words. stars: AGB and post-AGB - stars: variables: general - stars: individual: IRC+10216 - stars: mass-loss stars: circumstellar matter - techniques: high angular resolution

\section{Introduction}

Low- and intermediate-mass stars lose a large amount of their initial mass when they evolve along the Asymptotic Giant Branch (AGB) and beyond. During these mass-loss events, a huge circumstellar envelope (CSE) is formed. IRC+10216 is the best-known example of such evolved stars with an optically thick CSE. Indeed, its envelope almost completely absorbs the central stellar photons in visible light and at shorter wavelengths. This circumstellar environment has therefore been mostly studied in the infrared and millimeter domains, spectral regions where the envelope radiates itself and scatters the stellar light.

On very small scales (arcsec and below), a detailed picture of the IRC +10216 central regions has already been presented by several groups (see e.g. Haniff \& Buscher 1998; Weigelt et al. 1998, 2002; Tuthill et al. 2000, 2005). The central core appears to be composed of a series of clumps whose positions and luminosities vary on time-scales of a few years. The complexity of the detected structures has led to several hypotheses about the precise location of the central star.

On much larger scales (up to arcmin), Mauron \& Huggins (1999, 2000, MH99-00 hereafter) have shown that the IRC +10216 CSE can also be studied in visible wavelengths if enough deep images are collected. The nebula brightness then

$\star$ Based on observations collected with the VLT/Antu and Yepun telescopes (Paranal Observatory, ESO, Chile) using the FORS1 and NACO instruments (programs ID 63.I-0177A, 70.C-0565A, 70.C-0271B, 70.D-0271B). results when galactic ambient light is scattered by its dust particles. It is detected up to very large distances from the central star (up to about 6000 stellar radii), so it carries information about the mass-loss history during the last few thousand years. MH99-00 have also shown that this fairly round circumstellar envelope is consistent with an isotropic galactic radiation field and a spherically symmetric dust shell (see also Mauron et al. 2003). However, with a better spatial resolution $(\sim 1 \operatorname{arcsec})$, the envelope consists of a series of discrete and nested multiple shells (or arclets) whose origin is still debated. Although IRC+10216 is the only known AGB with such shells, similar morphology has already been detected around a dozen other planetary nebulae (PN) and about six proto-planetary nebulae (PPN). However, all these PN and PPN are bipolar, contrary to what we observe for their progenitor (assuming that IRC+10216 CSE properties are common to AGB stars). The cause and occurrence of the transition from a spherical multiple-shell CSE to a bipolar one is crucial to understanding the mass-loss phenomenon on the AGB and the evolution of the material ejected into the interstellar environment.

To date, no global view exists of the morphology of the IRC+10216 CSE at different scales. The aim of this work is to provide such a global description by combining new images of high dynamic range and high spatial resolution of its innermost regions, collected with adaptive optics techniques, together with new deep images of its most external layers. These observations are presented in Sect. 2. We analyze the morphology of the innermost regions and their temporal variations in Sect. 3. Section 4 
is devoted to analyze the numerous shells found in this envelope and some of their properties. We then discuss, in Sect. 5, the coexistence of the different morphologies found in the CSE of IRC+10216. Finally, a conclusion is presented in Sect. 6 .

\section{Observations and reductions}

\subsection{NACO observations}

Infrared images of IRC +10216 were recovered from the ESO Science Archive Facility. They were obtained in November 2002 and March 2003, using the adaptive optics system NACO at the ESO/VLT Yepun telescope. NACO is an association of the adaptive optics system NAOS (Rousset 2000) and the spectro-imager CONICA (Lenzen 2003).

We recovered observations of IRC +10216 obtained with the narrow-band filters NB 1.24 (centered at $\lambda_{\mathrm{c}}=1.237 \mu \mathrm{m}$, $\Delta \lambda=0.015 \mu \mathrm{m}), \mathrm{NB} 1.26\left(\lambda_{\mathrm{c}}=1.257 \mu \mathrm{m}, \Delta \lambda=0.014 \mu \mathrm{m}\right)$, $\mathrm{NB} 1.64\left(\lambda_{\mathrm{c}}=1.644 \mu \mathrm{m}, \Delta \lambda=0.018 \mu \mathrm{m}\right), \mathrm{NB} 1.75\left(\lambda_{\mathrm{c}}=\right.$ $1.748 \mu \mathrm{m}, \Delta \lambda=0.026 \mu \mathrm{m})$, NB $2.17\left(\lambda_{\mathrm{c}}=2.166 \mu \mathrm{m}, \Delta \lambda=\right.$ $0.023 \mu \mathrm{m})$ and the broad-band filter $L^{\prime}\left(\lambda_{\mathrm{c}}=3.80 \mu \mathrm{m}, \Delta \lambda=\right.$ $0.62 \mu \mathrm{m})$. The pixel scale on CONICA was 13.27 mas in the narrow-band filters and 27.15 mas in the $L^{\prime}$ filter. Observation conditions, as well as total on-source integration time for each filter, are summarized in Table 1. Calibration files (flat fields and dark exposures) and observations of the PSF reference star HR 3550 were also recovered. The Jitter technique was used in all observations. The box size of the $L^{\prime}$ broad-band image was about $7^{\prime \prime} \times 7^{\prime \prime}$, and the box sizes in the narrow-band images varied between about $4^{\prime \prime} \times 4^{\prime \prime}$ and $7^{\prime \prime} \times 7^{\prime \prime}$. As shown in Table 1 , the seeing conditions were variable, ranging between about $0.5^{\prime \prime}$ and $0.8^{\prime \prime}$. The best dynamic ranges of the IRC +10216 final images (not deconvolved) were around 7000, 40 000, 6000, and 90000 AFU (arbitrary flux units) for the $J, H, K$, and $L$ bands, respectively. The noise level was found to be smaller than 30 AFU in all images. In the PSF observations, the seeing varied between $0.5^{\prime \prime}$ and $0.6^{\prime \prime}$ and the air-masses between about 1.3 and 1.4. The estimated $F W H M$ of the PSF star was around 70 mas in the $J$ and $H$ bands, 80 mas in the $K$-band, and 120 mas in the $L$-band.

Standard reduction procedures were applied using selfdeveloped routines. The raw images were sky subtracted, then divided by the flat-field and corrected from hot pixels. In each filter, the images were cross-correlated and aligned by sub-pixel shifting, and then combined to produce the final images, eliminating cosmic rays hits. Finally, they were deconvolved with the PSF reference star. We used the Richardson-Lucy algorithm (Richardson 1972; Lucy 1974). Since no PSF data for IRC +10216 were found in the November 22, 2002 observations, we developed pseudo-PSF images for that night by analyzing and comparing the other IRC+10216 observations with their corresponding PSF data. Constancy of prominent features present in deconvolved images showed that the PSF selection and the number of iterations ( 25 typically) for the deconvolution process was performed carefully and conservatively. We then summed the deconvolved images in each band (see Fig. 1). The $J$-band image of highest dynamic range was obtained from the $1.24 \mu \mathrm{m}$ and $1.26 \mu \mathrm{m}$ images, which leads to about 14000 AFU. For the $H$-band, we summed both $1.64 \mu \mathrm{m}$ and $1.75 \mu \mathrm{m}$ narrow-band images, obtaining a dynamic range of about 78000 AFU. The three $2.17 \mu \mathrm{m}$ narrow-band images were combined to build a $K$-band image $(\sim 16000$ AFU). Finally, the $L$-band image has about 92000 AFU. These images have thus the best dynamic ranges ever published (see e.g. Tuthill et al. 2005). We note that,
Table 1. NACO observations log of IRC+10216.

\begin{tabular}{lccccr}
\hline \hline Date & Filter & $\begin{array}{c}\text { On-source } \\
\text { exp. time } \\
(\mathrm{s})\end{array}$ & $\begin{array}{c}\text { Seeing } \\
\left({ }^{\prime \prime}\right)\end{array}$ & $\begin{array}{c}\text { Air- } \\
\text { mass }\end{array}$ & $\begin{array}{r}\text { Dyn. } \\
\text { range } \\
\text { (AFU) }\end{array}$ \\
\hline 22 Nov. 02 & NB 1.64 & 128 & 0.6 & 1.5 & 28700 \\
& NB 2.17 & 120 & 0.6 & 1.6 & 6200 \\
16 Mar. 03 & NB 1.26 & 120 & $<0.5$ & 1.3 & 6900 \\
& NB 1.64 & 70 & $<0.5$ & 1.3 & 8300 \\
& NB 2.17 & 60 & $<0.5$ & 1.3 & 4800 \\
18 Mar. 03 & NB 1.24 & 210 & 0.6 & 1.3 & 7200 \\
& NB 1.75 & 103 & 0.7 & 1.3 & 41500 \\
& NB 2.17 & 200 & 0.8 & 1.3 & 5200 \\
& L $^{\prime}$ & 183 & 0.6 & 1.3 & 92500 \\
\hline
\end{tabular}

over the interval of about 4 months between the first and last observations studied here, no clear variations of the positions of the structures were found.

\subsection{FORS1 observations}

The observations were collected with the VLT-Antu telescope, equipped with the FORS1 focal reducer. The detector is a $2048 \times$ 2048 thinned $24 \mu \mathrm{m}$ pixel Tektronix chip. The field of view of individual images is $6.8^{\prime} \times 6.8^{\prime}$ with a pixel size of 0.2 arcsec (see Appenzeller et al. 1998). All the exposures were acquired in standard FORS1 service mode using a classical Bessel $V$-band filter. The available data consists of eight 10-min exposure and two 20-min exposure frames collected from 10 to 11 January 2000 , leading to a total observing time of $2 \mathrm{~h}$. The selected exposures were taken in dark time under very good seeing conditions and photometric sky. A few other 10-min frames were indeed rejected due to their moderate seeing conditions. The mean airmass was 1.3 . The telescope was shifted by a few arcseconds between individual images.

It was found that individual images reduced by the standard ESO reduction pipeline (includes standard corrections such as bias subtraction, flat fielding, etc., see Hanuschik \& Amico 2000) were of rather poor quality. We suspect this was due to the use of a corrupted flat field. Therefore, a new reduction procedure was performed for all individual exposures (removal of cosmic and aberrant pixels, flat-fielding with a specific mean sky flat for each night, etc.). All exposures taken on the same night were then shifted and stacked. The final reduced image was built by adding the summed exposures collected during the same night, accounting for their respective total exposure time. It consists of $1900 \times 1900$ pixels corresponding to a total field of view of $6.3^{\prime} \times 6.3^{\prime}$. The resulting mean seeing, measured from the brightness profile of individual stars, was found to be around $0.65^{\prime \prime}$. The central core of IRC +10216 was measured with a $S / N$ ratio larger than 100 per pixel and the $S / N$ of the envelope at $20^{\prime \prime}$ from the center is around 5-6 per pixel. For a more detailed description of this reduction procedure see Vandame (2002), and a preliminary presentation of this image can be found in de Laverny (2003). Due to the wide field of this image, we estimated a PSF reference by using the median average of a set of suitable pointlike stars, that were first background-subtracted, centered with sub-pixel accuracy, and normalized. The final FORS1 image (see Sect. 4) was deconvolved using a Richardson-Lucy algorithm (Richardson 1972; Lucy 1974). 

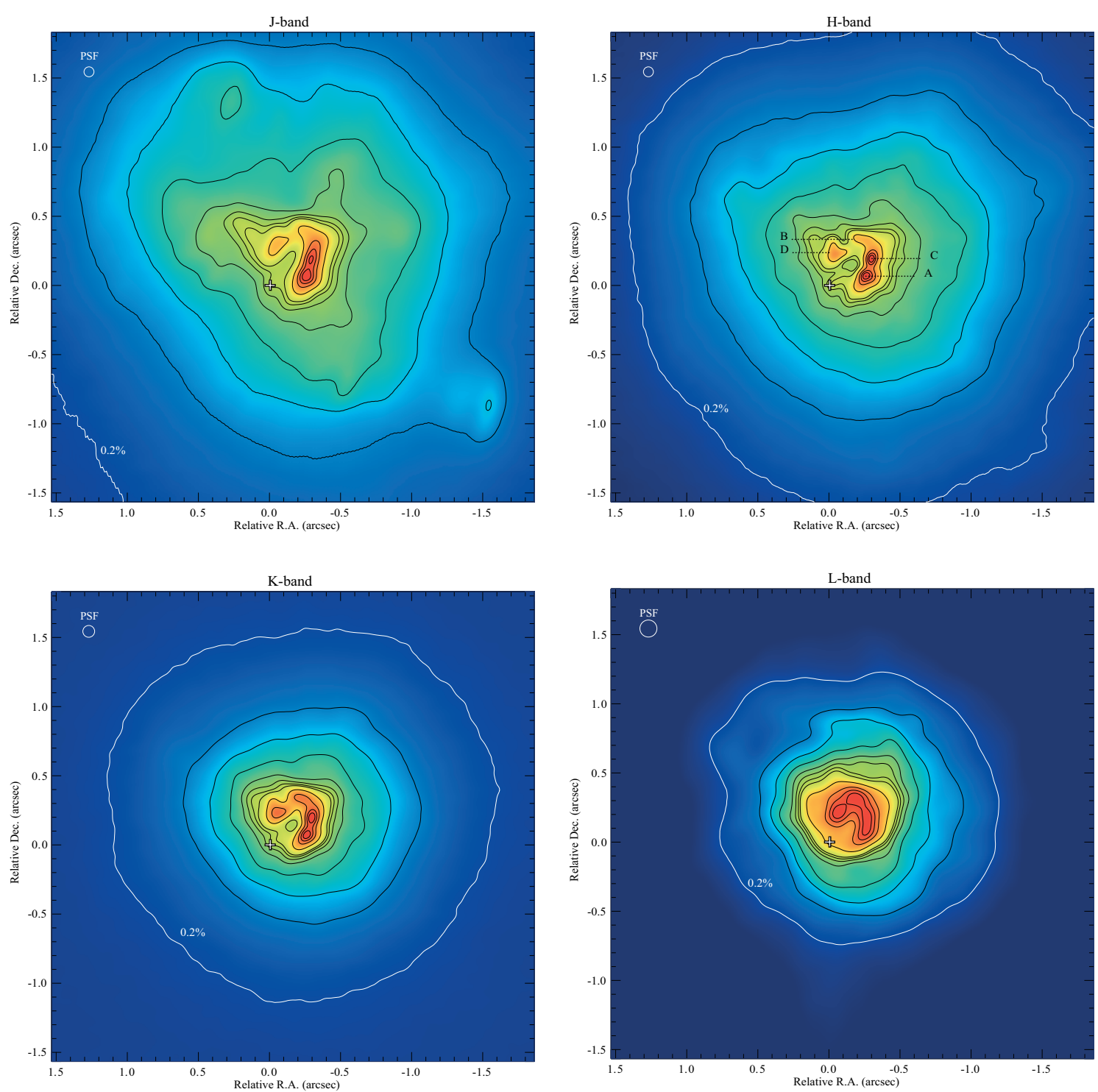

Fig. 1. NACO $J H K L$ images of IRC+10216. Contour levels are $80,60,40,20,10,8,6,4,2,1,0.5$, and $0.2 \%$ of the peak surface brightness. North is up and East is left. The white cross at each image center represents the assumed central star position and its size is proportional to the error of $0.03^{\prime \prime}$ as given by Murakawa et al. (2005). Clumps A to D of the $H$-band follow the Haniff \& Buscher (1998) clump nomenclatures. The resolution is about 70 mas in the $J$ and $H$ bands, 80 mas in $K$, and 120 mas in $L$ (represented by the circles at each image corner).

\section{The CSE innermost regions}

The JHKL diffraction-limited images of IRC+10216 are displayed in Fig. 1 with a log-scale for the brightness, so that details of the morphology at all flux levels can be seen. The labels A to $\mathrm{D}$ shown in the $H$-band image indicate the features identified and labeled by Haniff \& Buscher (1998). The images have been centered at the central star location estimated by Murakawa et al. (2005). These authors have performed a polarimetric study of IRC +10216 in $\mathrm{H}$-band that independently provides a possible central star position, after a series of contradictory hypotheses (see Weigelt et al. 2002 and Tuthill et al. 2005). Following Murakawa et al. (2005), we used clump A as a reference to identify the central star position. We note that their observations were made at the same epoch as the images presented here.

\subsection{Morphology at different wavelengths}

The images exhibit a bright and inhomogeneous structure that looks roughly like a ring (with a diameter of approximately $0.5^{\prime \prime}$ ) composed of clumps (including clumps A to D) around an approximately circular depression. The depression is located at about $(0.15,0.15)^{\prime \prime}$ from the image center and has about $6 \%$ of the intensity peak. Clumps $\mathrm{A}$ and $\mathrm{C}$ are the brightest features. Clump B appears as an elongated feature, at about the NE direction from clump $\mathrm{C}$, and clump $\mathrm{D}$ seems to be a more spread-out feature. In addition, there is a faint and extended envelope, almost spherical (from $\sim 0.5^{\prime \prime}$ to more than $1^{\prime \prime}$ from the image center), which also appears to have its center in the ring depression. This depression could thus correspond to an apparent center of the images. The central star is located in the fainter $\mathrm{SE}$ region of this ring. Its position also coincides with a particular elongation in the ring brightness distribution, which is very clearly seen in the $H$-band image. Finally, we verified that the faintest structures seen in the $J$-band are ghosts, probably due to the light reflected in the NACO optics.

On the other hand, a clear difference between the JHKL images concerns the brightness variations of clumps A to D with respect to the image peaks. Clump A remains close to the intensity 
peak in all bands, whereas clumps B and D are brighter at longer wavelengths (from about $10 \%$ and $20 \%$, respectively, of the intensity peak in $J$ to about $100 \%$ in $L$ ). The brightness of clump C increases more slightly and is always brighter than $80 \%$. We also note that the brightness difference between the four clumps strongly decreases with increasing wavelength. The SE region of the ring, close to the assumed location of the central star, remains faint, varying from about $8 \%$ to $20 \%$ of the intensity peak from $J$ to $L$. Finally, the extent of the external envelope seems to decrease with increasing wavelength. Considering its limits at $0.2 \%$ of the image peak brightness, we calculated its mean extent to be about $4.8^{\prime \prime}, 3.5^{\prime \prime}, 2.7^{\prime \prime}$, and $2.0^{\prime \prime}$ in the $J-, H-, K$-, and $L$-bands, respectively.

The clump brightness variations, as well as the extent of the envelope at different wavelengths, may reveal that we mostly detect, in $K$ and $L$, the emission of the heated dust, whereas at shorter wavelengths the scattered stellar emission becomes more important. This agrees with models of the spectral energy distribution computed for IRC+10216 (see e.g. Mauron et al. 2003). In the $K$ and $L$ bands, the dust emission is indeed $\sim 100$ times larger than the scattered stellar light, which becomes dominant below $\sim 1 \mu \mathrm{m}$. Therefore, we can deduce that most of the clumps seen close to the star in the $L$-band have approximately the same temperature. In contrast, optical depth effects in the $J$-band could explain the difference in brightness of the clumps.

\subsection{Temporal variations}

Temporal changes of the innermost regions of IRC +10216 have already been reported by Tuthill et al. (2000), Weigelt et al. (2002), and references therein. Weigelt et al. (2002) estimate approximately linear displacements between clumps A-C and A-D,

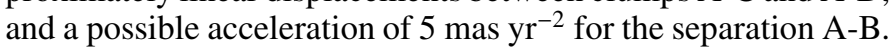
Tuthill et al. (2000, 2005) identify two sub-components in clump B: NE1 and NE2 close and far from clump C, hereafter referred to $\mathrm{B}_{1}$ and $\mathrm{B}_{2}$, respectively. They propose that clumps $\mathrm{B}_{1}$, $\mathrm{B}_{2}$, and $\mathrm{D}$ move away from $\mathrm{A}$, apparently with an uniform acceleration of 3.4 mas $\mathrm{yr}^{-2}$.

We also applied a Fourier filtering procedure on the $H$-band ${ }^{1}$ image in order to remove the structures of lower spatial frequencies (see Fig. 2). We then identified new features, in particular, the sub-features $\mathrm{B}_{0}^{\prime}, \mathrm{B}_{1}^{\prime}$, and $\mathrm{B}_{2}^{\prime}$ in clump $\mathrm{B}$. We found a feature, not identified in previous works, close to the assumed star position (labeled $\mathrm{H}$ ). We note that the star position assumed in this work could still be discussed and its connection with clump $\mathrm{H}$ is very unclear. This clump could be, for instance, a dust cloud just passing between the star and the observer. Future observations are needed to study the evolution of this clump with respect to the central star position.

Figure 2 shows estimated apparent trajectories for the previously detected clumps $\mathrm{B}_{1}, \mathrm{~B}_{2}$, and $\mathrm{D}$ with respect to $\mathrm{A}$. These estimations were made by assuming that the clumps move away from A, as proposed by Tuthill et al. (2000), and by taking their spatial separations into account. The solid arrows represent the displacement of these clumps during the interval of their observations, i.e. from January 1997 to April 1999. The dashed arrows show a prediction for the clump displacements up to March 2003 by assuming the averaged velocity of Tuthill et al. (2000). The

1 We selected the $H$-band image (instead of the $K$-band image, which has been most commonly analyzed) because of its better spatial resolution and considerably better dynamic range. We verified that the detected clumps and their estimated positions are similar to those found in the $K$-band.

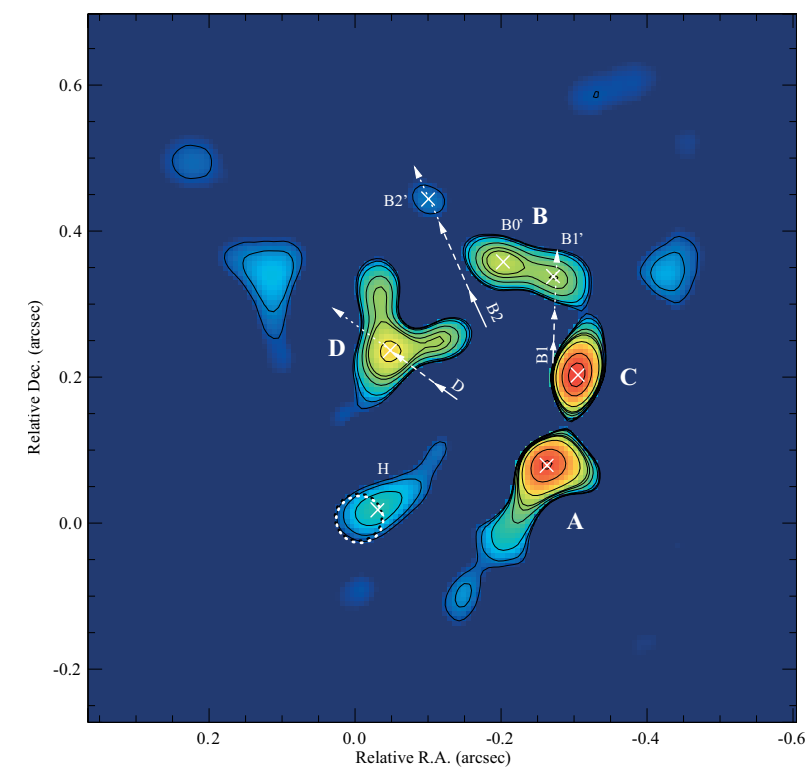

Fig. 2. $H$-band map of IRC+10216, where only the highest spatial frequencies of the brightness in the Fourier space have been kept. The contour levels are $80,50,20,10,8,5,2,1$, and $0.5 \%$ (this minimum level being the estimated noise). The dotted circle is the assumed star position, as in Fig. 1. Main clumps are indicated by the labels A to D and some sub-features by the smaller labels. The positions of these clumps are shown by the white crosses. Estimated trajectories (from the results of Tuthill et al. 2000) for some clumps with respect to clump A are also shown (see text for details).

dotted arrows represent an alternative prediction by assuming the acceleration law proposed by Tuthill et al. (2000). The error margins are about 10 mas for the dashed arrows and 30 mas for the dotted ones. We can see that the previous clumps $\mathrm{B}_{1}$ and $\mathrm{B}_{2}$ are most probably the current sub-features $\mathrm{B}_{1}^{\prime}$ and $\mathrm{B}_{2}^{\prime}$, respectively. They are currently separated by $258 \pm 20,394 \pm 20$, and $261 \pm 20$ mas from $A$. Thus $B_{1}$ and $B_{2}$ appear to be less accelerated than expected. At the same time, clump D appears to have moved with a constant velocity. From the clump separations given by Weigelt et al. (2002), we also verified that clump C (currently located at $131 \pm 20$ mas from A) appears to be nearly motionless. Note that choosing clump A as a reference could give the illusion that the clumps escape from it. The clump motions are therefore not compatible with the uniform acceleration law proposed by previous studies, although some accelerations may exist for clumps $\mathrm{B}_{1}$ and $\mathrm{B}_{2}$. New high angular-resolution observations are needed to disentangle the three-dimensional morphology of the innermost environment of IRC+10216 and to study the temporal variations of these clumps.

\section{External layers of IRC+10216}

Figure 3 shows the deconvolved wide-field $V$-band image of IRC +10216 . As already shown by MH99-00, we see an extended halo composed of thin and irregular multiple-shells. They appear to be non-concentric and azimuthally incomplete. The CSE is seen due to external illumination by the ambient Galactic light, scattered by the dust. Since these photons can easily penetrate into the CSE (their optical depth from the outside towards the center being very low), the incomplete shells do reveal lower densities in some parts of the CSE. Obviously, the shell discontinuities cannot be caused by some shadowing effects due to more external material. 


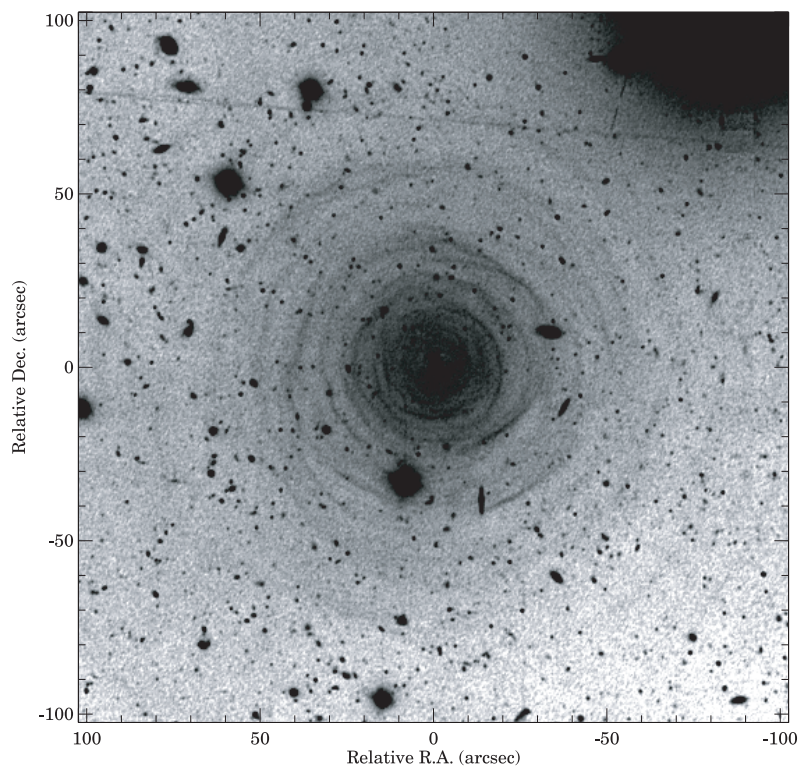

Fig. 3. FORS1 deconvolved $V$-band image of IRC +10216 . North is up and East is left.

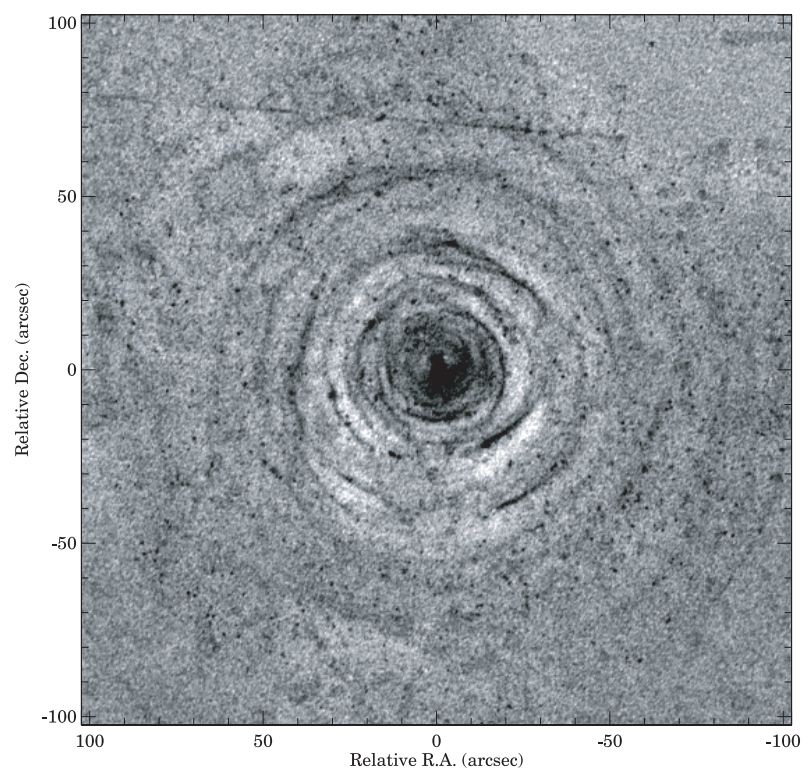

Fig. 4. Deconvolved $V$-band image after subtraction of the halo of the CSE and removal of most stars and galaxies.

\subsection{Structure of the external layers}

To emphasize the shell morphology, we removed the central extended halo by applying the same Fourier filtering procedure as for the NACO images. We also removed several sources (stars or galaxies) by selecting those with observed intensities larger than a prefixed threshold. The source pixels were replaced by averaged values taking the local background and the noise level into account. The resulting image is shown in Fig. 4. We then applied an azimuthal smoothing of $20^{\circ}$ around the center. Although this decreases the spatial resolution in the azimuthal direction, the resulting map (Fig. 5) shows a clear visualization of the shells and gives a more realistic and complete pattern than that presented in previous works.

The shell distribution seen in Figs. 4 and 5 are similar to those reported before. For instance, the three faint and apparently thick shells at North, located at about 30-40", 50-60",

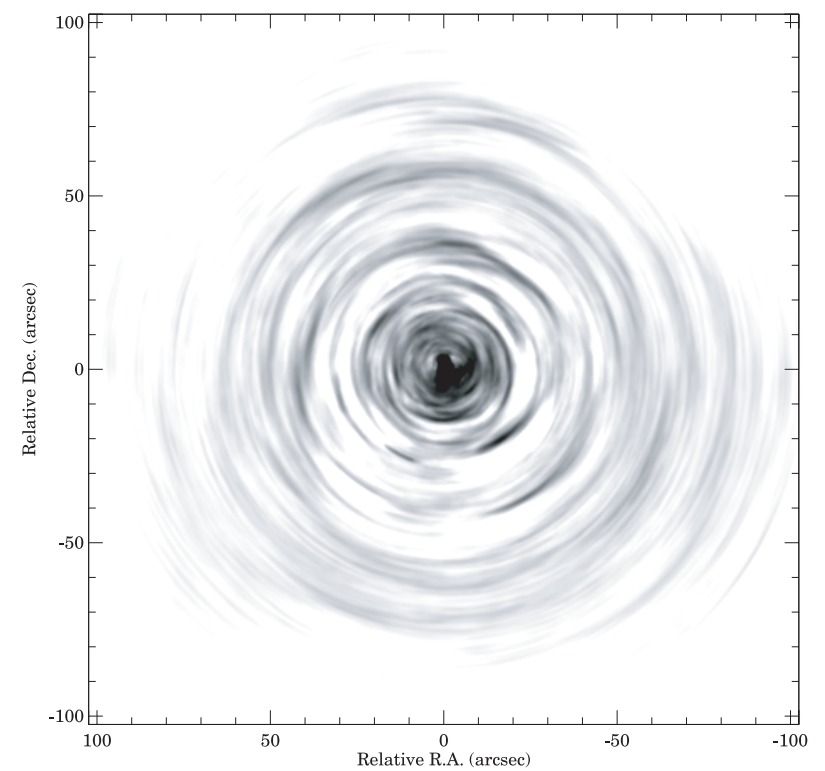

Fig. 5. Schematic map of the shells surrounding IRC+10216 (see text for details).

and 70-80" from the center, can also be seen in the CFHT image (MH99-00). However, these shells, being better resolved in the FORS1 images, appear to be composed of a complex subdistribution of thinner ones. Similar thin shells located very close each other are clearly seen everywhere in the CSE. For instance, the shells labeled $e$ and $f$ by MHOO (located at distances from the center of about $55^{\prime \prime}$ and $58^{\prime \prime}$, between $347-20^{\circ}$ and $23-53^{\circ}$, respectively) are clearly mergers of complex thin-shell distributions. Another prominent shell located to the $\mathrm{S}$ at about $15^{\prime \prime}$ from the center joins series of slightly less prominent thinner ones distributed toward SE between 10-30" from the center. Moreover, even in the more internal regions, several thin shells seem to merge in thicker ones between about $4^{\prime \prime}$ and $20^{\prime \prime}$. The whole CSE thus appears to be composed of a complex of several thin irregular shells that could be identified as thicker ones in less resolved images. Finally, we note that the separation between apparently thick shells varies a lot with respect to the radial direction.

On the other hand, a smooth azimuthally radial profile derived by computing the mean of all the pixels found in annuli $0.9^{\prime \prime}$ thick (see MH99-00 for more details) confirms that the dust is detected up to about 200" (about 5800 stellar radii). That corresponds to material ejected about 8000 years ago (assuming an escape velocity of $14 \mathrm{~km} \mathrm{~s}^{-1}$ and a distance of $120 \mathrm{pc}$ ), i.e. an important fraction of an interpulse on the AGB. Actually, we do not see any edge for the dusty envelope, and we are limited by the detector size.

\subsection{Thickness of the shells}

MH00 propose that the shell thickness increases when increasing the distance from the center, in agreement with the natural expansion of the envelope. To analyze the shell profiles and to verify their finding, we propose here a new and more accurate method, by taking into account the non-concentric nature of the shells and the possibility that an apparently thick shell may be resolved into several thinner ones. 

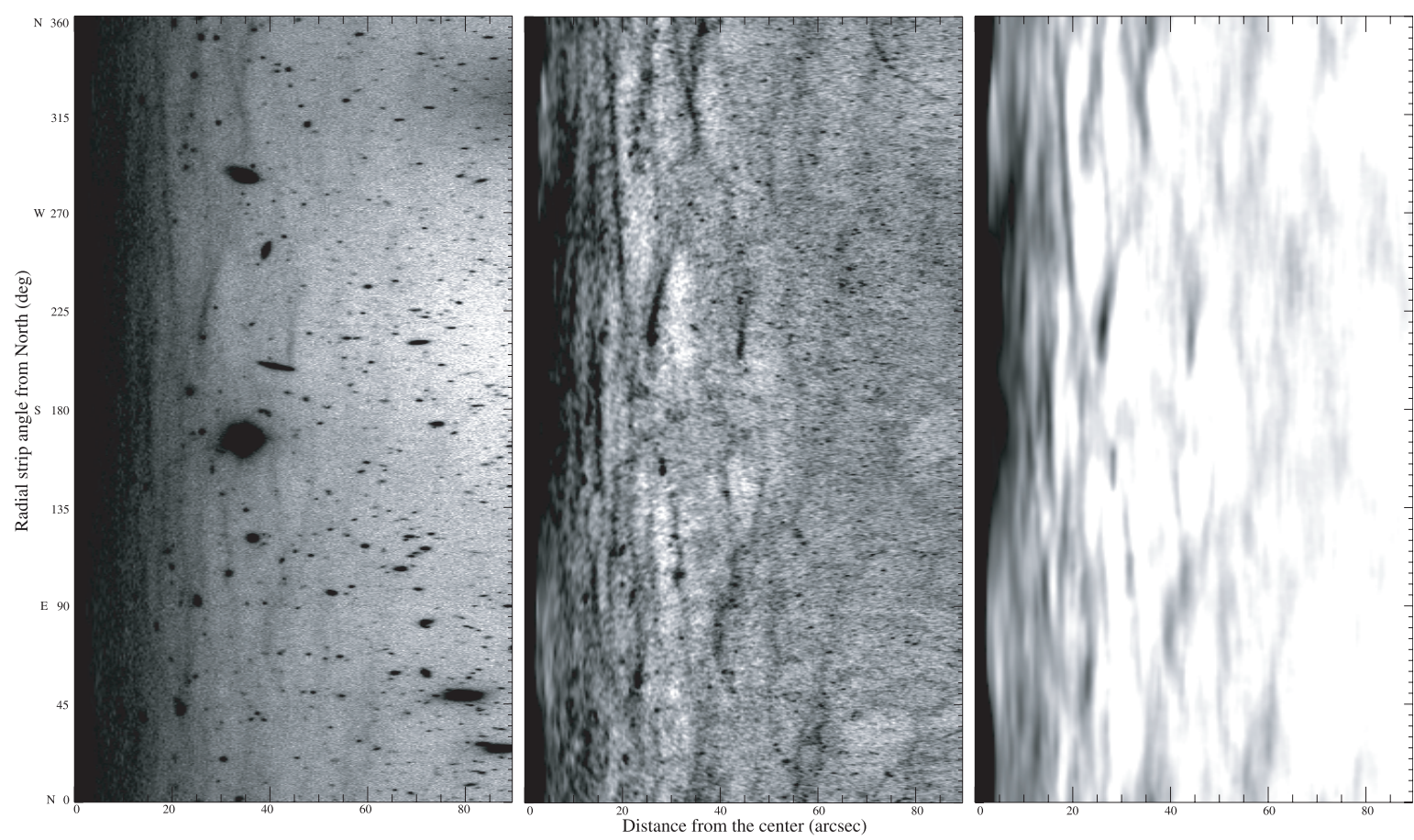

Fig. 6. Map of the shells transformed from polar coordinates to a Cartesian representation. Each horizontal section of these maps represents a narrow radial strip of the FORS1 images, the angles of the strips being with respect to North. Maps to the left, center, and right were derived from Figs. 3, 4 and 5, respectively.

Figure 6 shows the CSE morphology in a map of narrow ${ }^{2}$ radial strips. From this diagram, we clearly see the non-concentric nature of the shells, as well as the complexity of their spatial distribution. For instance, the long and thin shell located at distances from the center of about $15-20^{\prime \prime}$, between $220^{\circ}$ and $340^{\circ}$ from North, has an inclination of about $4^{\circ}$ in this diagram with respect to the vertical axis. The shell located at distances from the center of about $30-35^{\prime \prime}$, between $300^{\circ}$ and $360^{\circ}$ from North, has an inclination of about $17^{\circ}$. At the same time, there are shells with opposite orientations, such as those found at distances from the center of about 25-30" and 45", from $210^{\circ}$ to $260^{\circ}$, and from $200^{\circ}$ to $230^{\circ}$, respectively, which make angles of about $-16^{\circ}$ and $-2^{\circ}$ with respect to the vertical axis.

Regarding the profile of the thickest shells, we carefully analyzed the shell labeled $d$ in MH00. Figure 7a shows its profile by applying the same method as those authors. Figure $7 \mathrm{~b}$ shows the profile of the same shell estimated from the more restricted region located at a distance from the center of about $39^{\prime \prime}$, between $70-90^{\circ}$, where it appears more regular. We then integrated profiles perpendicular to its direction and subtracted the extended halo contribution. The estimated $F W H M$ of profile (a) is about 3.0" (as in MH00), whereas it is about 2.6" for profile (b). The error margins are around $0.4^{\prime \prime}$. Although both estimates agree within the error bars, a deeper analysis of this shell reveals that even our profile (b) could be widened due to a merging of two thinner ones. Indeed, the profiles shown in Fig. 7 have two close peaks at offsets of about $\pm 0.5^{\prime \prime}$, which leads us to suspect that there are two thin shells close together in this region, hence, not spatially well resolved. We note that this shell was carefully analyzed, this pair of peaks being identified in every derived profile. If we decompose profile (b) in two close shells, their estimated $F W H M$ are about $1.8^{\prime \prime} \pm 0.4^{\prime \prime}$. In consequence,

${ }^{2}$ Radial sections with a thickness of 1 pixel and a rotation step of $0.2^{\circ}$. Interpolations between the original image pixels were applied at each step.

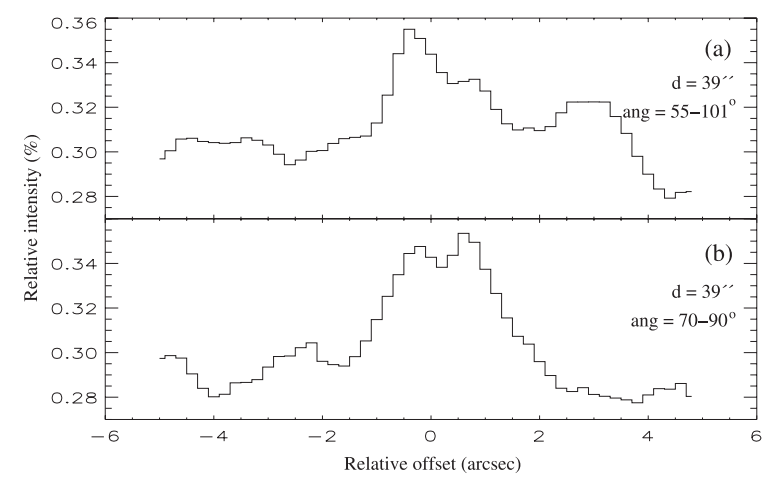

Fig. 7. Comparison of the profile of the shell $d$ estimated as in MHOO (top panel) with its profile derived by our more complex method (bottom panel, see text for details). The distances from the center, $d$, of the shells and the ranges of their azimuthal angles from north, ang, are given. The relative intensity is with respect to the central peak brightness of the original image.

we derived several shell profiles by identifying, as above, wellresolved thin shells at different distances from the center (see Fig. 8). Profiles (a) to (d) have good $S / N$ ratios. Profile (e) with a lower $S / N$ ratio is actually a thin feature composing an apparently thicker shell that was also detected by MHO0. The FWHM of the two features in profile (a) and the other four features in profiles (b) to (e) are, respectively, about: 1.2", 1.6", 1.5", $1.7^{\prime \prime}, 1.6^{\prime \prime}$, and $1.4^{\prime \prime}$. The error margins are around $0.4^{\prime \prime}$. In a more general way, we estimated the $F W H M$ of 23 shells at radial distances from $4^{\prime \prime}$ to $80^{\prime \prime}$ (see Fig. 9). We found a mean $F W H M$ value of $1.6^{\prime \prime}$, with a standard deviation of $0.3^{\prime \prime}$. The minimum $F W H M$ value of $1.2^{\prime \prime}$ was found for the innermost analyzed shell, located at about $4^{\prime \prime}$ from the center between $40-100^{\circ}$, and also for two shells located at about $9^{\prime \prime}$ and $11^{\prime \prime}$ from the center, between $150-180^{\circ}$ and $205-255^{\circ}$, respectively. The maximum $F W H M$ value of $2.0^{\prime \prime}$ is found for the shells located at about $24^{\prime \prime}$ and $25^{\prime \prime}$, between $105-135^{\circ}$ and $60-85^{\circ}$, 


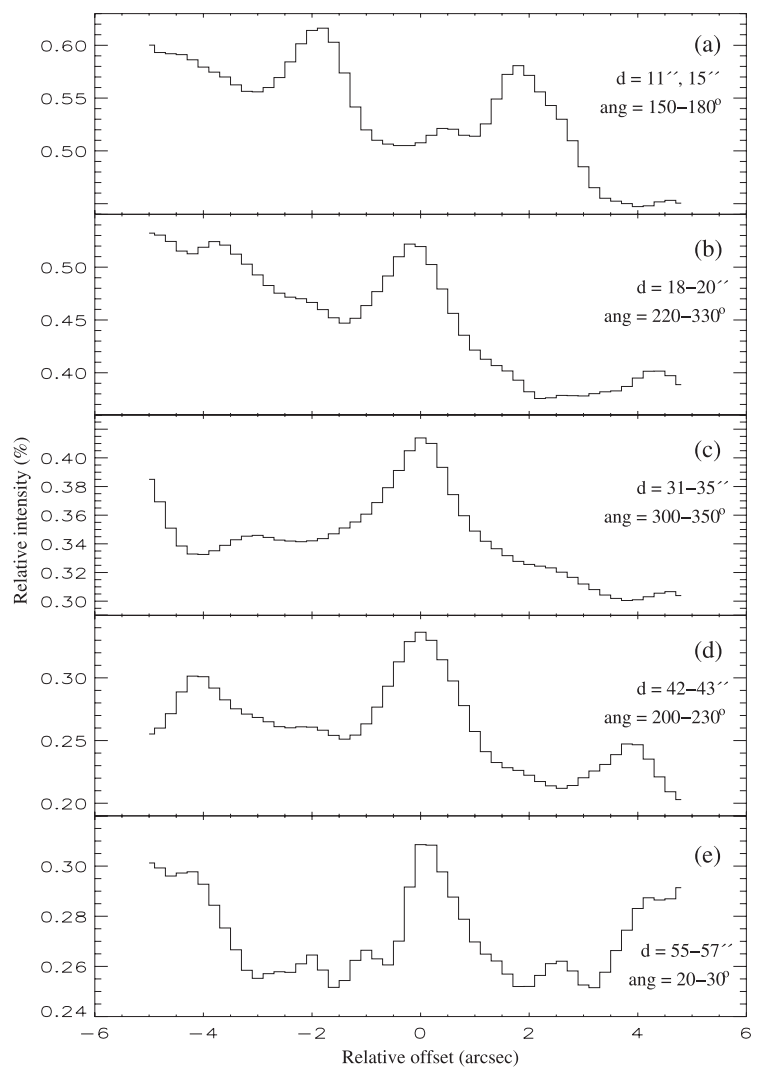

Fig. 8. Profiles of some shells considering their non-concentric nature (see text for details). There are two shells in panel (a) and one in the others. The given parameters follow the same definitions as in Fig. 7. The ranges in the shell distances from the center, $d$, are due to their non-concentric nature.

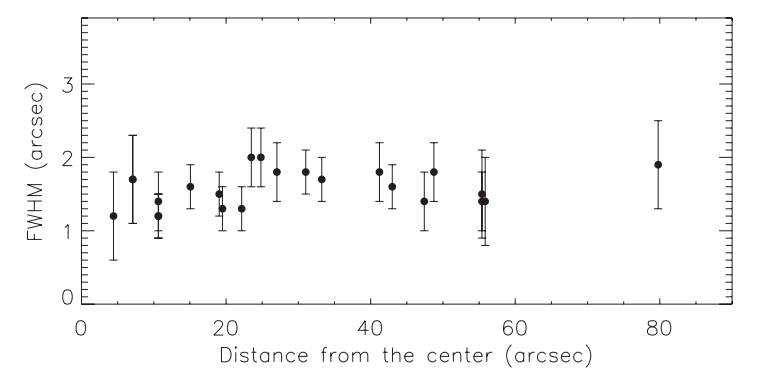

Fig. 9. Relation between the thickness of the shells and their distance from the center.

respectively. The furthest analyzed shell, located at about $80^{\prime \prime}$ from the center, between $30-45^{\circ}$, has $F W H M \simeq 1.9^{\prime \prime}$. The error bars vary between $0.3^{\prime \prime}$ and $0.6^{\prime \prime}$. Thin shells are thus detected in the whole envelope and even far from the center. We therefore cannot derive a clearly increasing relation between the shell thickness and the distance from the center such as proposed by MH00. However, the shells found rather close from center could be resolved into even thinner ones. We therefore could have overestimated their thickness. In consequence, either the slope of the thickness variation with distance proposed by MH00 could still be valid but with very thin shells close to the star, or the shell thickness increases much less than estimated by these authors.

\section{Global view of IRC +10216}

In order to better understand the possible links existing between the almost spherical shells and the inner clumps, we describe here the morphology of the inner CSE from the FORS1 image together with the NACO data.

Figure 10 (left panel) shows a closer view of Fig. 4. MH99-00 have detected three structures suspected to be shells in regions within about 3.1" from the center, whereas no such shells are found in our data, possibly because the HST data have a better spatial resolution, although a lower $S / N$ ratio. The closest identifiable structures are located between $\sim 3^{\prime \prime}$ and $16^{\prime \prime}$ from the center. The core of the nebula appears clearly asymmetric. Two dominant lobes much brighter than the rest of the envelope lie around the center, together tracing a direction of about $22^{\circ} \pm 2^{\circ}$ with respect to North. The southern lobe is $40 \%$ brighter than the northern one. These features probably result from scattered stellar photons in contrast to the reflected galactic light seen elsewhere in the envelope. Such bipolar morphology could be an indication that scattering is more efficient in the polar direction. It could be roughly reproduced by a simple model of scattering dust grains in a non-spherical dusty envelope, with evacuated polar regions around the star, the system being tilted away from the observer (see e.g. Men'shchikov et al. 2001). We note, however, that the main shape of the bipolar nebula differs slightly from the one reported by MHOO from their HST image, possibly due to the spatial resolution.

Figure 10 (right panel) gives a representation of the IRC+10216 core by superposing the NACO and FORS images. The two images were arbitrarily positioned by coinciding their intensity peaks. We are aware that this assumption may be crude since the $\mathrm{V}$ and IR images stem from very different physical processes. However, this composite image represents, for the first time, both the extended layers and the bipolar core, together with the real size of the inner clumps, and makes it clear how difficult it is to find a link between such small and large scale morphologies. First, evidence of clumps far from the center was not found by Huggins \& Mauron (2002) in a previous analysis of the same FORS1 image. Second, shells cannot be identified in the NACO images. We note that, although the region composed of clumps A to D roughly looks like a ring, its center (the depression feature) is not compatible with the star position estimated by Murakawa et al. (2005). Finally, the bipolar structure detected in $V$-band is also not clearly identified in the near-IR.

\section{Conclusion}

We have described very high quality images of the CSE of IRC +10216 , from its most inner regions to the most external ones. In the central arcsec scale of the JHKL images, sub-arcsec structures (or clumps) identified by other authors were recovered about 4 years later. We have also derived a map of the brightest clumps found close to the core of IRC +10216 . The morphology of these clumps varies a lot with increasing wavelengths, and we propose that the closest structures have about the same temperature. Furthermore, by analyzing their apparent relative motion, we cannot confirm the uniform outflow acceleration previously proposed, since only two bright clumps appear to be accelerated (but at a lower rate than that already estimated), whereas others clumps could have a constant velocity or even no relative motion. On much larger spatial scales (up to a few arcmin), we present a new map of the non-spherical incomplete shells characterizing the CSE of IRC+10216. Owing to the high spatial resolution of our image, most of the thicker shells actually appear to be composed of thinner elongated ones. Their thicknesses appear rather uniformly distributed between about $1^{\prime \prime}$ and $2^{\prime \prime}$, regardless of the distance from the center. Finally, we combined the NACO and FORS images in order to provide a more global view of this 

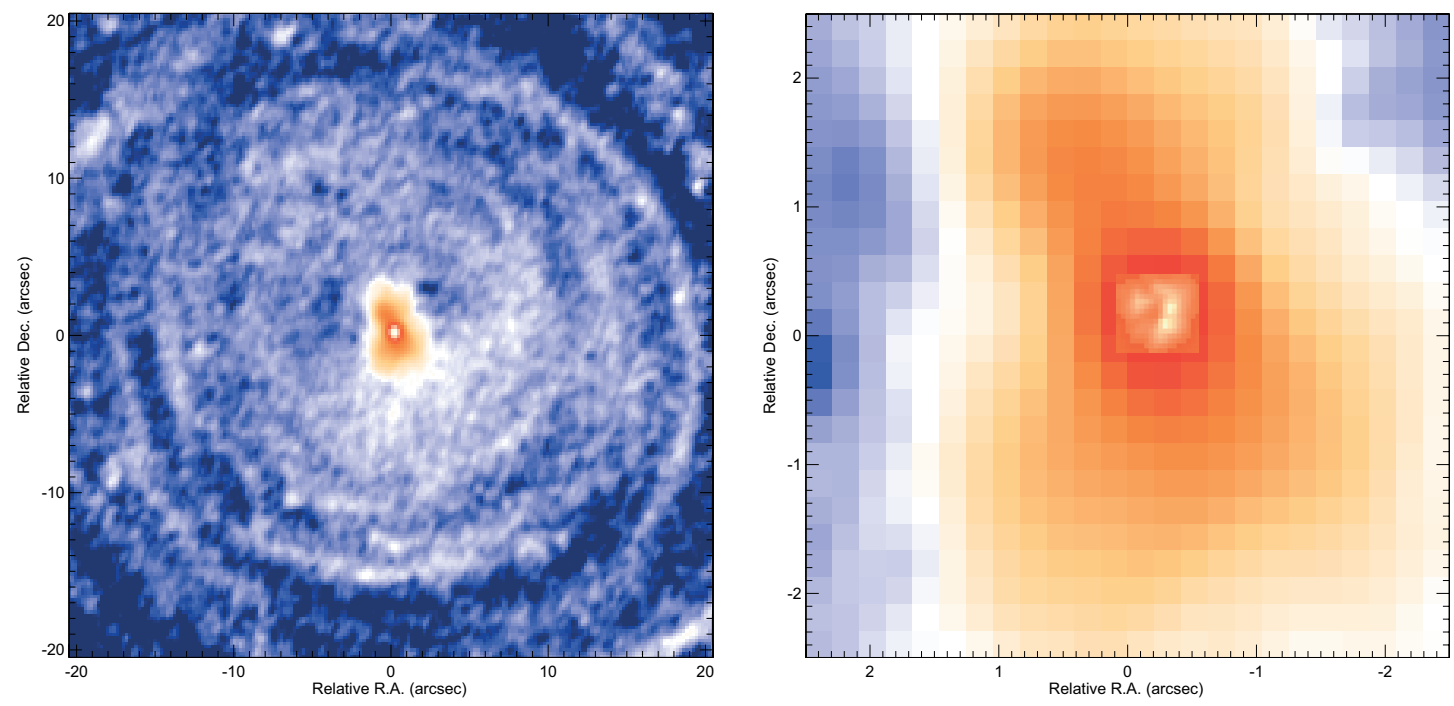

Fig. 10. View of the IRC+10216 CSE inner morphology. The left panel shows the closest structures around the center detected in $V$-band. The right panel shows the $V$-band image core, on which the NACO $H$-band image has been superposed. The overlapping assumes that the NACO and FORS peak brightness are found at the same location.

CSE and to compare the typical size of the clumps found very close to the center with the bipolar nebula and with the much more external shells.

This study has confirmed the very complex nature of the IRC +10216 envelope with asymmetries already present on the AGB. Neither the morphology at different spatial scales nor the motions detected very close to the center can be satisfactory explained by current models of the mass-loss mechanisms in AGB stars and their typical time-scales. For instance, Steffen \& Schönberner (2000) have studied the formation of such discrete shells and shown that short episodes of high mass-loss could translate into a narrow, high-density shell moving through the envelope. They also propose an alternative mechanism resulting from the interaction of a faster inner wind running into a slower outer one. However, very few shells seem to be produced by such mechanisms, and their mutual distance cannot be easily connected to the stellar evolution or stellar variability on the AGB. On the other hand, considering a more consistent model, Sandin \& Höfner (2004, and references therein) predict shell density distributions that are not compatible with those observed around IRC +10216 . Another scenario for the formation of the shells in a spherically symmetric stellar wind has been explored by Soker $(2000,2002)$. He proposes that these shells could be connected to cool magnetic spots on the stellar surface. If these spots are more concentrated near the equator, the massloss geometry could deviate from sphericity and thus favor the formation of shell-like features and/or clumps.

Moreover, it is interesting to note that the very complex structures found around IRC+10216 may affect the chemical composition of its envelope. For instance, the clumps detected very close to the central core may favor, by their thermodynamical properties, the formation of the graphite observed in presolar dust grains (Bernatowicz et al. 2005). Furthermore, the presence of high density shells in the photochemically active regions could change the molecular distribution in the envelope by blocking external UV photons (see e.g. MH00; Brown \& Millar 2003). Then, high contrast shells of complex molecules may be formed more easily, as confirmed by some millimeter observations (see for instance, $\mathrm{HCO}^{+}, \mathrm{C}_{2} \mathrm{H}, \mathrm{C}_{4} \mathrm{H}$, and $\mathrm{HC}_{5} \mathrm{~N}$ maps by Guélin et al. 2000, and CO maps by Fong et al. 2003).
Finally, future high-spatial resolution images of this CSE are still mandatory in order to better understand the motions of its clumps (and in particular of clump $\mathrm{H}$ superposed on the assumed central star), their formation/fading, the central star position, and the possible evolution of the external shells as their three-dimensional morphology.

Acknowledgements. We thank N. Mauron for fruitful and stimulating discussions through all these years and for his comments on the manuscript, and D. O'Brien for proofreading it. The Brazilian agencies CAPES and $\mathrm{CNPq}$ are thanked for financial support. P. de Laverny acknowledges support from the CNRS/INSU (Actions Thématiques Innovantes) and MESR (Jeunes Chercheurs).

\section{References}

Appenzeller, I., et al. 1998, The Messenger, 94, 1

Bernatowicz, T. J., Akande, O. W., Croat, T. K., et al. 2005, ApJ, 631, 988

Brown, J. M., \& Millar, T. J. 2003, MNRAS, 339, 1041

Fong, D., Meixner, M., \& Shah, R. Y. 2003, ApJ, 582, L39

Guélin, M., Lucas, R., Neri, R., et al. 2000, in IAU Symp., 197, 365, ed. Minh, \& van Dishoek

Haniff, C. A., \& Buscher, D. F. 1998, A\&A, 334, L5

Hanuschik, R., \& Amico, P. 2000, The Messenger, 99, 6

Huggins, P. J., \& Mauron, N. 2002, A\&A, 393, 273

de Laverny, P. 2003, in Mass-losing pulsating stars and their circumstellar matter, ed. Y. Nakada, M. Honma, \& M. Seki, Astrophysics \& Space Sience Library, 283, 197 (Kluwer Academic Press)

Lenzen, R., Hartung, M., Brandner, W., et al. 2003, SPIE, 4841, 944

Lucy, L. B. 1974, AJ, 79, 745

Mastrodemos, N., \& Morris, M. 1999, ApJ, 523, 357

Mauron, N., \& Huggins, P. J. 1999, A\&A, 349, 203

Mauron, N., \& Huggins, P. J. 2000, A\&A, 359, 707

Mauron, N., de Laverny, P., \& Lopez, B. 2003, A\&A, 401, 985

Men'shchikov, A. B., Balega, Y., Blöcker, et al. 2001, A\&A, 368, 497

Murakawa, K., Suto, H., Oya, S., et al. 2005, A\&A, 436, 601

Richardson, W. H. 1972, JOSA, 62, 55

Rousset, G., Lacombe, F., Puget, P., et al. 2000, SPIE, 4007, 72

Sandin, C., \& Höfner, S. 2004, A\&A, 413, 789

Simis, Y. J. W., Icke, V., \& Dominik, C. 2001, A\&A, 371, 205

Soker, N. 2000, ApJ, 540, 436

Soker, N. 2002, ApJ, 570, 369

Steffen, M., \& Schönberner, D. 2000, A\&A, 357, 180

Su, K. Y. L. 2004, ASPC, 313, 247

Tuthill, P. G., Monnier, J. D., Danchi, W. C., et al. 2000, ApJ, 543, 284

Tuthill, P. G., Monnier, J. D., \& Danchi, W. C. 2005, ApJ, 624, 352

Vandame, B. 2002, SPIE, 4847, 123

Weigelt, G., Balega, Y., Blöcker, T., et al. 1998, A\&A, 333, L51

Weigelt, G., Balega, Y. Y., Blöcker, T., et al. 2002, A\&A, 392, 131 